\title{
Insights into breast cancer phenotying through molecular omics approaches and therapy response
}

\author{
Jose E. Belizario' ${ }^{1}$, Angela F. Loggulo \\ 'Department of Pharmacology, Institute of Biomedical Sciences, University of São Paulo, Avenida Lineu Prestes, 1524, São Paulo, \\ CEP 05508-900, Brazil. \\ ${ }^{2}$ Department of Pathology, Paulista School of Medicine, Federal University of São Paulo, Rua Sena Madureira, 1500, São Paulo, \\ CEP 04021-001, Brazil.
}

Correspondence to: Prof. Dr. Jose E. Belizario, Department of Pharmacology, Institute of Biomedical Sciences, University of Sao Paulo, Avenida Lineu Prestes, 1524, Sao Paulo, CEP 05508-900, Brazil. E-mail: jebeliza@usp.br

How to cite this article: Belizario JE, Loggulo AF. Insights into breast cancer phenotying through molecular omics approaches and therapy response. Cancer Drug Resistance 2019;2:527-38. http://dx.doi.org/10.20517/cdr.2018.009

Received: 18 Dec 2018 First Decision: 8 Mar 2019 Revised: 15 Apr 2019 Accepted: 27 Jun 2019 Published: 19 Sep 2019

Science Editor: Enrico Minin Copy Editor: Cai-Hong Wang Production Editor: Jing Yu

\begin{abstract}
Breast cancer is the most common cancer in the world. Despite advances in early detection and understanding of the molecular bases of breast cancer biology, approximately $30 \%$ of all patients with early-stage breast cancer have metastatic disease. Breast cancers are comprised of molecularly distinct subtypes that respond differently to pathway-targeted therapies and neoadjuvant systemic therapy. However, no tumor response is observed in some cases and development of resistance is most commonly seen in patients with heterogeneous breast cancer subtype. To offer better treatment with increased efficacy and low toxicity of selecting therapies, new technologies that incorporate clinical and molecular characteristics of intratumoral heterogeneity have been investigated. This short review provides some examples of integrative omics approaches (genome, epigenome, transcriptome, immune profiling) and mathematical/computational analyses that provide mechanistic and clinically relevant insights into underlying differences in breast cancer subtypes and patients'responses to specific therapies.
\end{abstract}

Keywords: Breast cancer, ERBB/HER, estrogen receptor, progesterone receptor, genomics, proteomics, epigenomics, endocrine and targeted therapy

\section{INTRODUCTION}

Cancer is defined as genetic disease and is molecularly characterized by accumulation of mutations and epimutations that lead to functional dysregulation of cell genome and epigenome-driven processes ${ }^{[1]}$.

Cc) (7) The Author(s) 2019. Open Access This article is licensed under a Creative Commons Attribution 4.0 International License (https://creativecommons.org/licenses/by/4.0/), which permits unrestricted use sharing, adaptation, distribution and reproduction in any medium or format, for any purpose, even commercially, as long as you give appropriate credit to the original author(s) and the source, provide a link to the Creative Commons license, and indicate if changes were made.

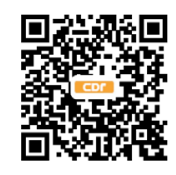


Nonetheless, different genetic and epigenetic variations within patients to patients can lead to a same disease phenotype, e.g., uncontrolled cell growth. Recent advances in whole genome throughput DNA sequencing, systems biology and machine learning algorithms have produced promise information on the evolutionary dynamics of tumors, from a single cell to a cell population ${ }^{[2-4]}$. The complex landscape of somatic modifications (copy number variations, mutations, gene rearrangements) observed in breast tumors are typically the result of a relatively small number of functional driver oncogenic alterations and a large number of non-functional passage alterations. The functional consequence of most cancer mutations could be characterized in phenotypic readouts such as growth assays or drug response screens using tumor cell lines ${ }^{[5-7]}$. The impact of mutations on kinase binding specificity has been explored in vitro protein binding microarrays, or in vivo, for example, by measuring kinase target phosphorylation after kinase mutation ${ }^{[8]}$. However, what substantially contribute to oncogenesis and progression of tumors is intra and inter-clonal heterogeneity, which is determined by a stochastic mutational process in cancer cells $s^{[9,10]}$.

Most common factors taken into account for classification of tumors into distinct groups include: degree of local invasion, degree of remote invasion, histological types of cancer and specific grading based on various tumor markers, and general status of the patient ${ }^{[2,3]}$. However, cancers with similar morphological and histopathological features reveal very distinct patterns of progression and response to therapy. With growing number of database of tumor and single cell genomic, epigenomic, and pharmacogenomics reporting on differences and similarities between clinical outcomes of patient cohort to the same therapies, we have a rewarding opportunity to modeling and predicting outcomes to traditional chemotherapy and targeted therapies ${ }^{[11]}$.

Epigenetic alterations participate in all steps of cancer, from tumor initiation to cancer progression and metastasis ${ }^{[1,12]}$. It is now well understood that both losses and gains of DNA methylation occurring in CpGs motifs, as well as aberrant chromatin organization, contribute significantly to cancer associated phenotypes ${ }^{[12]}$. Epigenetic inactivation of tumor suppressor genes (TSGs) is a well-established event in cancer progression. Oncogenic activating mutations are now known to occur in a number of epigenetic modifiers such as isocitrate dehydrogenase 1 and 2, histone-lysine $\mathrm{N}$-methyltransferase enzyme (EZH2), DNA methyl transferases (DNMTs ${ }^{[13]}$. In cancer cells, global DNA hypomethylation is frequently concomitant with both local hypo and hypermethylations (epigenetic mosaicism). This may contribute to cancer progression through chromosomal instability, reactivation of transposable elements and loss of imprinting. The DNMTs: DNMT1, DNMT2, and the DNMT3A/3B/3L are main components methylation machineries whereas demethylases Ten-eleven translocation proteins TET1, TET2 and TET3, and repressive histone-modifying enzymes of the Polycomb repressive complex 1 (PRC1) and PRC2, specifically named, "writers" and "erasers" are modulators of epigenetic patterns ${ }^{[12]}$. Defects in DNA methylation could induce TSG silencing and resistance to classical chemotherapeutic agents. Interplay between DNA methylation and DNA repair machineries may also occasionally provoke de novo methylation and aberrant gene silencing ${ }^{[12-14]}$. Methylome profiles provides a molecular signature of cancers and might serve as potential diagnostic and predictive biomarkers ${ }^{[1-17]}$. Some new epigenetic modifier drugs combined with conventional chemotherapies and immunotherapies may reverse more permanent changes that affect the epigenetic processes such as histone hypermethylation and transcriptional gene silencing ${ }^{[18]}$.

Genomic and post-genomic research technologies have shifted to focus on biomarker discovery for diagnosis, prognosis and prediction of treatment response to targeted therapies ${ }^{[6]}$. Continuous discovery of cancer subtypes has proven that tumor cell surface markers used in traditional pathology-based classifications and clinical phenotyping cannot capture the full complexity of tumors ${ }^{[2-4]}$. In tumors, different cell types interact with each other in the tumor microenvironment (TME). Immune and stroma cells of TME play an important role in tumorigenesis and development of metastasis ${ }^{[9,10]}$. TME studies revealed that tumors can be classified into inflamed and non-inflamed tumors ${ }^{[19]}$. The first type contains 
abundant presence of immune cells: T cells, myeloid cells, monocytes, and the second little or no presence of immune cells, especially $\mathrm{T}$ cells ${ }^{[19]}$. Fibroblast cell types are part of TEM, and more precisely cancerassociated fibroblasts (CAFs) have pro-tumor functions in breast cancer as they can enhance metastasis ${ }^{[20]}$. The presence of tumor-infiltrating lymphocytes (TILs) in TME is associated with an overall patient good prognosis, better survival and the success of checkpoint immunotherapy ${ }^{[19]}$. Studies performed a multiomic analysis of Tumor Cancer Genome Atlas (TCGA) datasets have allowed identification at least six immune subtypes across cancer types ${ }^{[21]}$. Finally, stromal cells and immune cells can preserve the properties of cancer stem cells (CSCs), or cancer initiating cells, which are cells that exert multicellular functions in tumor tissue-specific networks and immune resistance ${ }^{[22,23]}$. More important, CSCs display differentiation-state plasticity that allow cancer cells to undergo epithelial to mesenchymal transition (EMT), a process in which cancer cells acquire migratory and invasive properties ${ }^{[24]}$. These results underline the importance of immunophenotyping as a new modality to sub-classify cancers based on their $\operatorname{TME}^{[19,20]}$.

The effectiveness of the targeted therapy strongly depends on both the cancer type and molecular features of the individual tumors ${ }^{[25,26]}$. The context-specific impact of molecular features such as somatic alterations and/or copy number events can be measured using diverse high-throughput techniques such as transcriptomics (the number of counts of mRNA molecules) and (phospho) proteomic and transcription factor (TF) activities ${ }^{[27,28]}$. The reverse phase protein array (RPPA) is a high-throughput antibody-based technique, similar to Western blot, to evaluate protein activities in signaling networks ${ }^{[27,28]}$. This functional proteomic analysis can be done in either flash-frozen or formalin-fixed, paraffin-embedded (FFPE) tissue samples. The use of RPPA data for evaluation of functional signatures linking perturbations in down- and up-stream signal transduction pathways might be crucial for personalizing cancer therapies in future ${ }^{[18]}$.

Computational integrative methods that combine genomic and functional cancer phenotypes may better predict those patients who will benefit of the combination therapies ${ }^{[27,28]}$. This system biology approach generally uses statistical/mathematical modeling and supervised machine learning for learning and predict disease similarities from basic and clinical data. Personalized disease subnetworks may be necessary to uncover cancer-related associations, including genotype-phenotype relationships and spatial heterogeneity in the tumor microenvironmental interactions ${ }^{[4,27,28]}$. However, although powerful, the use of these methodologies still requires additional strategies to reveal functionally important biomarkers, which often remains the rate-limiting step in the diagnostic challenge. Here we will discuss these issues using as model the breast cancer tumors.

\section{BREAST CANCER SUBTYPES AND THERAPY OUTCOMES}

Breast cancer has the highest incidence in women worldwide and is the fifth leading cause of mortality in the globe. Many breast cancer classifications have been proposed according to the invasive characteristics, occurrence, histology and molecular profiling of tumor samples ${ }^{[29,30]}$. Based on their site of occurrence, tumors can be classified as lobular (located at breast lobules) or ductal (at breast ducts). Carcinomas may also arise from invasive epithelial cells (medullary carcinoma), mucus-producing cells (mucinous carcinoma, also called colloid carcinoma), or a subtype of ductal carcinoma in situ (DCIS) or invasive ductal carcinoma (tubular carcinoma). The in situ to invasive breast carcinoma progression is often caused by interactions among epithelial, myoepithelial, and stromal cells. The progression occurs due to the loss of normal myoepithelial cell function ${ }^{[3]}$.

Cancers derived from luminal cells are the most common types of breast cancer expressing hormone receptors for estrogen receptor (ER), progesterone receptor (PR), or the amplified human epidermal growth factor receptor (EGFR) 2/erythroblastic leukemia viral oncogene homolog 2 receptor (HER2/ 
ErbB2/ERBB2 $)^{[30]}$. A high resolution copy-number analyses have confirmed recurrent amplification on chromosomal regions and genes, respectively, found in primary tumors mapping at 8q24 [v-myc myelocytomatosis viral oncogene homolog (avian) (MYC)], 11q13 [cyclin D1 (CCND1)], 17q12 (ERBB2), $20 \mathrm{q} 13$ [serine/threonine kinase 15 (STK15)/aurora kinase A], and homozygous deletion at 9p21 [cyclin dependent kinase inhibitor $2 \mathrm{~A}(\mathrm{CDKN} 2 \mathrm{~A})]^{[32]}$. Tumors lacking expression of all three receptors (ER, PR, HER2) are referred to as triple-negative breast cancers (TNBCs), which are tumors most often derived from cells of basal origin. TNBCs display stem cell-like and luminal progenitor-like gene signatures, and frequently have somatic mutations in the TSGs TP53 and PTEN, and a smaller fraction is also mutant for breast cancer 1 (BRCA1). Molecular gene expression profiling has also redefined breast cancer subtypes as luminal A, luminal B, HER2-rich, and basal-like, which roughly parallel the immune-histochemical categories $^{[33-35]}$. The basal and claudin-low subtypes map to the previously designated basal $\mathrm{A}$, and basal B subtypes, respectively ${ }^{[36]}$. In general, the luminal A breast cancer subtype displays mutations in phosphatidylinositol 3-kinase (PIK3CA) ( 49\%), mitogen-activated protein kinase (MAP3K1) ( 14\%), GATA binding factor $3(14 \%)$, TP53 ( 12\%), and MAP2K4 ( 12\%) and loss of Phosphatase and tensin homolog deleted on chromosome ten (PTEN) (13\%), among others. The luminal B breast cancer subtype has mutations in TP53 ( 32\%), PIK3CA ( 32\%), MAP3K1 ( 5\%) and other genes. HER2-overexpressing tumors display representative TP53 mutation and overexpression of other genes, such as growth factor receptorbound protein 7 (GRB7) and Post-GPI Attachment To Proteins 3. Patients with HER2-overexpressing tumors usually develop brain metastases and additional mutations in fibroblast growth factor receptor 2, PI3KCA and ataxia telangiectasia and rad3-related kinase, homozygous deletion in CDKN2A as well as amplification in Kirsten rat sarcoma viral oncogene homolog $(\mathrm{KRAS})^{[33-36]}$. Breast cancer patients with BRCA1 germline mutation do not express ER, PR, and HER2 and share morphological, clinical, and molecular features and immunohistochemical and cytokeratin expression patterns like basal like breast cancers ${ }^{[30,37]}$.

Immunohistochemistry and/or fluorescence in situ hybridization in slides or tissue microarray (TMA) have been used to identify the distinct primary and invasive and non-invasive breast cancer subtypes ${ }^{[30]}$. The subtyping is critical in clinical management of distinct prognoses and predictive responses to endocrine or targeted therapy ${ }^{[38,39]}$. Breast cancers with HER2/ERBB amplification respond to trastuzumab and/or lapatinib, and tumors with mutated or amplified BCR-ABL (breakpoint cluster region C-ABL oncogene 1 , non-receptor tyrosine kinase) respond to imatinib mesylate ${ }^{[40]}$. However, although molecular profiling provides important prognostic indicators, breast cancer risk stratification remains a challenge in TNBC cases $^{[41]}$. For instance, the claudin-low subtype was identified as a TNBC subset that is associated with more aggressive tumor behavior and worse prognosis ${ }^{[4]]}$.

Figure 1 shows the Kaplan-Meier curves that exemplify how hierarchical clustering based on patterns of expression of intrinsic genes or gene expression signatures can discriminate tumors and ultimately the outcomes following the adjuvant and combined systemic treatment ${ }^{[33]}$. Many independent data sets representing different patient cohorts have confirmed the discriminatory power of the molecular subtypes for the representative groups and recommended their treatment as separable diseases ${ }^{[33]}$. Diverse panels of well-characterized breast cancer cell lines have been used to statistically validate the robustness of associations between molecular subtypes and activated signaling pathways to in vitro specific therapeutic compound responses ${ }^{[5-7]}$. Surprisingly, more than 50,000 genetic and molecular features emerged from these cell lines which exhibit differential sensitivities to most therapeutic compounds $s^{[5-7]}$. Similar mutational profiles have been identified in tumor samples derived from patients'cohorts such as METABRIC (the Breast Cancer International Consortium), TCGA and many other cancer genome cohorts ${ }^{[42]}$. Analyses of subclonal evolution of a cancer cell across lifespan indicated that a dominant common ancestor lineage is present at time of tumor diagnosis ${ }^{[43]}$. Deep analyses of cancer genomes identified over 30 cancer mutational signatures, which are caused by multiple mutational processes include infidelity of either the DNA replication, damage or repair machinery ${ }^{[43]}$. At least six genome rearrangement signatures driving 


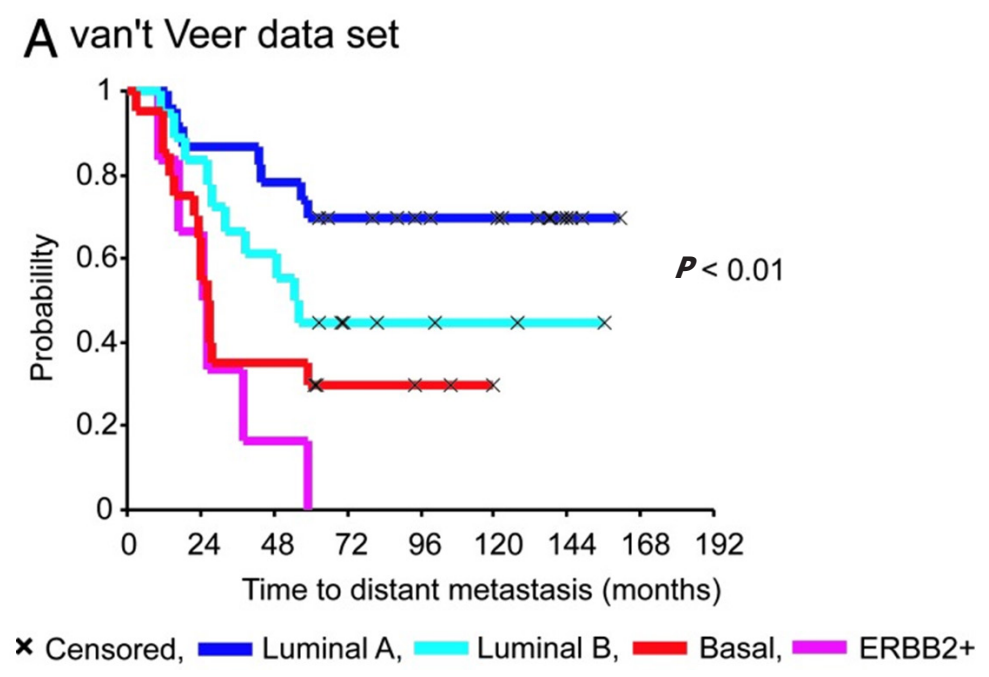

B Norway/Stanford data set

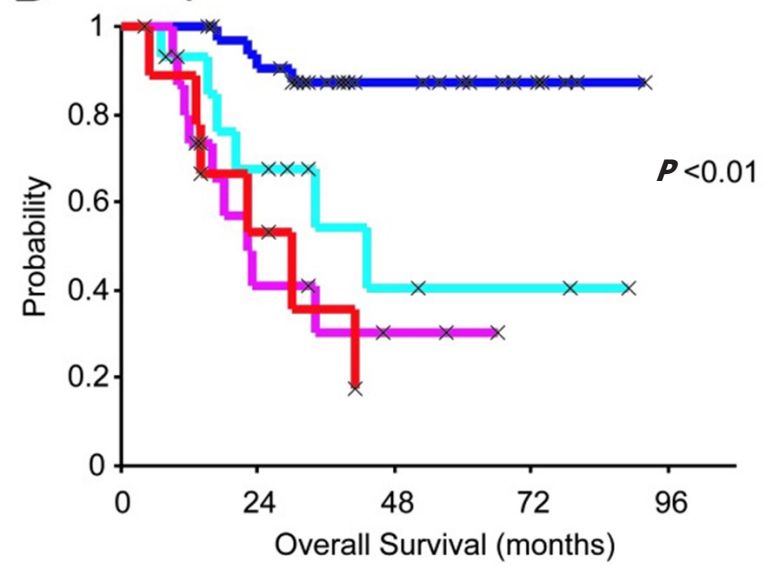

Figure 1. Kaplan-Meier curves of time for distant metastasis (A) and overall survival (B) among five breast cancer subtypes in two patient cohorts. This figure is quoted with permission from Sorlie et al. ${ }^{[33]}$

the genomic amplification have been identified in breast cancers ${ }^{[42]}$. These clinical and genomic datasets and somatic mutational catalogues are available online for in silico investigation for their relationships to tumor clinical responses ${ }^{[42,43]}$.

Growing evidence suggests that CAFs is the major cellular component in the peritumoral microenvironment and a strong biomarker for breast cancer growth invasion and dissemination ${ }^{[20,24]}$. CAFs display mesenchymal-like features and are likely mesoderm derived and differ from normal fibroblasts in terms of gene expression profile, activation mechanism and phenotype ${ }^{[44]}$. A study in a Brazilian cohort of breast cancer patients confirmed that CAFs in lymph nodes with macrometastasis express similar profile of vimentin, alpha-smooth muscle actin (-SMA), and S100A4 protein as those CAFs found in primary tumors. CAFs were uniformly ER, PR, HER2, MKI67, and P53 negative, but level of staining of transforming growth factor-1 (TGF-1), CXC chemokine receptor CXCR4, and p-AKT STK(62.3\%, 52.4\%, 65\%, respectively) were equivalent between primary and lymph node metastasis fibroblasts ${ }^{[4]}$. Some anticancer drugs are likely to affect CAFs, however none is known about the impact on therapeutic outcomes.

The presence of distinct global gene-expression signatures within tumors allowed the development of reliable omics-based technologies for clinical diagnostics of breast cancer subtypes ${ }^{[3-36]}$. The Oncotype 
Basal

Luminal B
HER-2 enriched
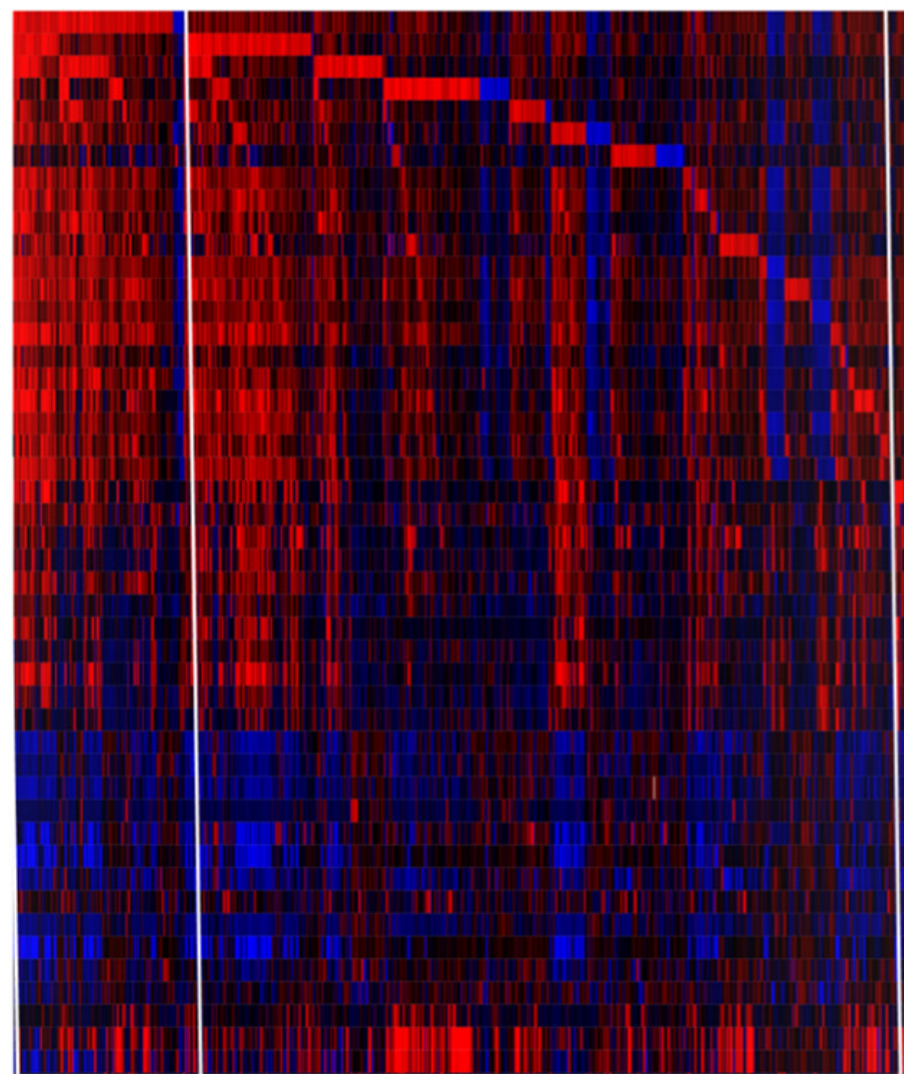

Luminal A

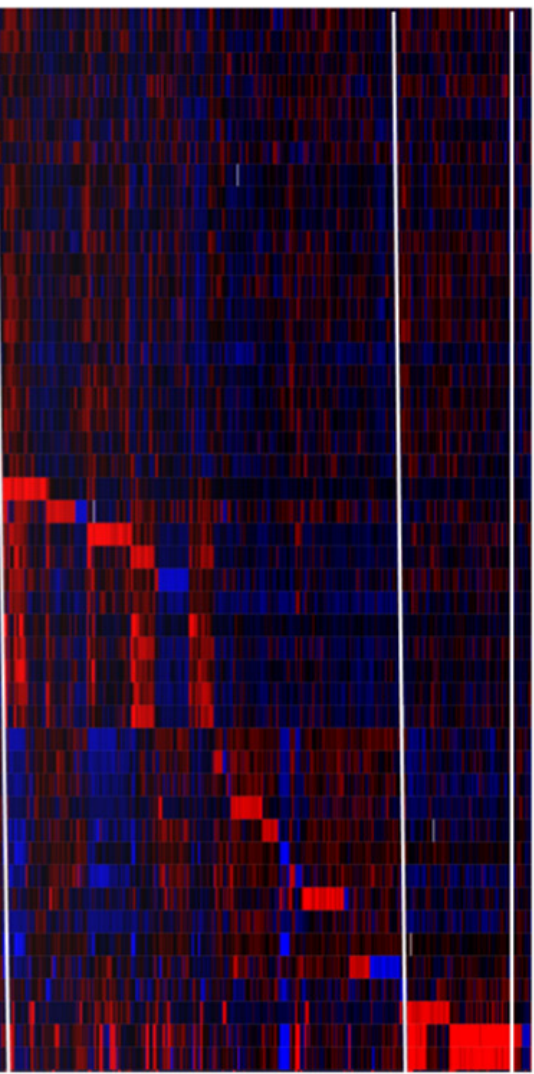

Figure 2. Heatmap of RNA expression (z-scores) of the PAM50 gene set in 1904 breast tumors samples from METABRIC cohort. The visual representation of clustered gene patterns associated with subtypes Basal, Luminal B, luminal A, HER-enriched and Normal-like (left to right order). Each rows correspond to a gene and each column to a patient sample. The data of BAG1, PPR160 and TMEM45B were not included. The heatmap was generated using cBioPortal tools (https://www.cbioportal.org/)

DX, PAM50, and MammaPrint tests are some examples commercially available ${ }^{[45]}$. The routine application of these tests are useful for identifying stage I or stage II lymph node-negative breast cancer patients that may or may not require adjuvant chemotherapy ${ }^{[30,46]}$. The Oncotype-DX breast cancer assay quantifies the transcription for only 21 genes through reverse transcription polymerase chain reaction using in the breast cancer tissue (lumpectomy, mastectomy, or core biopsy) conserved in FFPE. The OncotypeDX test provides a recurrence score (from 1 to 100) that is used to predict the risk of recurrence of DCIS breast cancer subtypes and/or the risk of a new invasive cancer ${ }^{[45]}$. The Oncotype DX DCIS test in woman diagnosed with DCIS predicts benefit from radiation after surgery. There is currently clinical practice guideline addressing when biomarker scores might be applied for predicting which women will benefit of specific drugs or regimens for adjuvant systemic therapy ${ }^{[45]}$.

The PAM50 test uses 50 gene expression set to discriminate between each of the 5 breast cancer subtypes. The PAM50 recurrence score estimate the risk of distant recurrence of hormone-receptor-positive breast cancer from 5 to 10 years after diagnosis and after 5 years of hormonal therapy treatment in postmenopausal women $^{[30,45]}$. For illustrative propose we present a heatmap generated in cBioPortal (https://www.cbioportal. org/) for PAM50 gene set in 1904 breast tumors samples from METABRIC study [Figure 2]. This colored representation displays similarities between the tumor samples in terms of messenger RNA expression levels (z-scores) for the panel of genes. The rows correspond to indicated genes and column to samples. The gene clusters that define subtypes Basal, Luminal B, luminal A, HER-enriched and Normal-like markers 
are separated from left to right order. The PAM50 scores incorporate most highly enriched clusters of genes associated with ER signaling (12 genes), growth factor signaling (4 genes), proliferation (21 genes), invasion (1 gene), basal phenotypes (9 genes) and miscellaneous (3 genes). Cytokeratins KRT5, KRT14 and KRT17, as well as basal markers SFRP1 and MIA differentiates Basal-like from Luminal B tumors. ERBB2, EGFR, FGFR4 and GRB7 differentiates HER2-enriched tumors from other subtypes. The PAM50 and Oncotype DX share the genes MKI67, CCNB1, BIRC5 and MYBL2, which contribute to their proliferation score. The prognostic value of PAM50 is greater than ER/HER2 immunohistochemistry classification ${ }^{[30,45]}$. However, studies have shown that PAM50 score did not improved outcomes of patients who underwent anthracyclineand taxane-based therapy ${ }^{[30,47]}$.

Many predictive modeling methodologies for phenotyping breast cancer subtypes have focused on signaling transduction upon the canonical signaling pathways, including PI3K, mammalian target of rapamycin, MAPK, transforming growth factor $\beta$, Wingless/Integrated, cell cycle, apoptosis, immune responsiveness, and DNA damage response pathways ${ }^{[3,27,28]}$. Protein phosphorylation, a reversible posttranslational modification at serine $(\mathrm{S})$, threonine $(\mathrm{T})$ and tyrosine $(\mathrm{Y})$ residues, involves a system of sequence-specific kinases (writers), phosphatases (erasers) and reader proteins. The advent of reverse RPPA databases has allowed the phophoproteomic profiling of key cancer-related proteins. The RPPA platform of the University of Texas MD Anderson Cancer Center (https://bioinformatics.mdanderson.org/main/ MCLP) displays the protein expression in 650 independent cell lines with known genomic, transcriptomic, and drug-screening data. Analyses in this dataset revealed mutation-induced perturbations of over 200 proteins and their phosphorylated forms in various cancer types ${ }^{[48]}$. The representative nodes and modules of cell network perturbations were used to determine specific clusters for each cancer type. For instance, the breast cancer cluster was distinct from other cancer types by high expression of proteins of androgen hormone receptor canonical pathway and HER2 protein ${ }^{[48]}$. RPPA-based pathway-activation profiling can be a powerful tool to predict putative mechanisms underlying sensitivity and resistance to breast tumor subtypes for specific therapy ${ }^{[48]}$. The phospho-HER2 and phospho-EGFR profiles are particularly important in clinical setting for prescription of EGFR pathway-targeted drugs such as Herceptin, lapatinib and pertuzumab ${ }^{[40]}$.

HER2/ERBB2 is a transmembrane glycoprotein belonging to EGF family of receptors that regulate cell growth, proliferation, survival, differentiation, and angiogenesis ${ }^{[40]}$. Activation of the ERBBs receptors by phosphorylation leads to complex signaling pathways ${ }^{[40]}$. GRB7 is one of the 105 protein coding genes located in the same amplicon as HER2 on chromosome 17q12. Over-phosphorylation of GRB7 in ERBB2 amplified tumors is involved in resistance to anti-HER2 and antiestrogen therapy. A comprehensive gene set enrichment analysis revealed perturbed ERBBs signaling in HER2 amplified breast cancer cells overexpressing dermcidin (DCD), a gene localized at chromosome 12q13 locus and potential oncogene of breast epithelial cells ${ }^{[49]}$. Agreeing with this postulated, we found that DCD is co-expressed with GRB7, ERBB2 and FGFR4 in various cohorts of breast tumor samples, including METABRIC cohort (data not shown). More importantly, DCD has been considered as biomarker for cellular resistance of various tumor cells to the EGFR/ErbB1 tyrosine kinase inhibitors erlotinib and lapatinib ${ }^{[50]}$.

Mutations in PIK3CA gene encoding the p110 $\alpha$ catalytic subunit of PI3K, class IA, are among the most common alterations in human malignancies and contribute to approximately $25 \%$ of breast cancers ${ }^{[30]}$. Such mutation confers a gain of function to $110 \alpha$ and resistance to HER2-based therapy ${ }^{[51]}$. A combination of three proteins comprising the receptors EGFR, ERBB3/HER3, and the cyclin-dependent kinase inhibitor p27 (CDKN1B) was found to be a potential biomarker for dependence on PI3K/AKT vs. MAPK/ ERK signaling for drug resistance in HER2 breast cancers ${ }^{[52]}$. A computational algorithm called affinity regression was developed for analysis of distinct dysregulated transcriptional regulators downstream of oncogenic somatic alterations ${ }^{[53]}$. The analyses and validation of the method was done across 12 TCGA 
cancer data ${ }^{[53]}$. The differential activity of TFs associated to mutant PIK3CA in isogenic breast cancer cell lines was revealed by comparing the phospho (p)-AKT and p-S6K levels ${ }^{[53,54]}$. The results point toward the possible application of these predictive models to screening of drugs to personalized therapies.

Epigenetic studies have found that luminal breast cancers share a common epigenetic signature, whereas basal-like breast cancers display highly heterogeneous signatures ${ }^{[55,56]}$. Basal-like breast cancer cells that constitute the majority of TNBCs can reprogram a subset of luminal breast cancer cells to a basal-like state, which drastically alter their phenotype. This phenotype is associated with high production of the cytokine IL-6 and tumor-promoting molecules ${ }^{[56]}$. Overexpression of three TFs Engrailed 1, T box transcription 18, and T-cell factor 4 can induce the repression of some luminal features in luminal breast cancer cell $\mathrm{s}^{[56]}$. Over $75 \%$ of breast tumors express the ER $\alpha$ that leads to genetic and cellular aberrations ${ }^{[38,39]}$. Patients with ER $\alpha$-dependent tumor growth often respond well to tamoxifen and fulvestrant ${ }^{[38,39]}$. Nonetheless, patients may develop endometrial cancer and increased bone density. Recent studies using ChIP-seq and chromatin accessibility (DNase-seq and ATAC-seq) assays have identified a unique cistrome ER $\alpha$ profile for breast cancers $^{[57]}$. The TF FOXA1 was found as an essential TF for estrogen binding and induction of ER $\alpha-$ mediated gene expression in breast cancer cells ${ }^{[58,59]}$. Therefore, targeting FOXA1 with small molecules may disrupt ER $\alpha$ positive breast tumor growth. The cyclin D and cyclin-dependent kinases 4 and 6 (CDK4/6) have critical roles in breast carcinogenesis ${ }^{[29,30]}$. Experimental chemotherapy with small molecule inhibitors of CDK/6, such as abemaciclib, palbociclib and ribociclib, have yielded clinical benefits to ER positive breast cancer patients ${ }^{[60]}$. Abenamaciclib decreases cell proliferation and enhances tumor cell intracellular levels of endogenous retroviral genes, triggering "viral mimicry". This in turn stimulates production of type III IFNs and IFN-induced genes in an autocrine fashion ${ }^{[60,61]}$. Accordingly, treatment of cancer cells with abemaciclib markedly decrease DNMT1 mRNA levels and DNA methylation, therefore, functioning in the similar way as epigenetic modifier 5 -azacytidine ${ }^{[62]}$. Finally, epigenetic changes at enhancers and promoters of breast cancer cells as well as tumor stroma cells such as CAFs and myoepithelial cells are critical for transformation and metastasis ${ }^{[20,24]}$. CAFs may also contribute to immune suppressive effect of TME that is one specific biological feature of HER2/neu-positive breast cancers ${ }^{[63]}$. Therefore, DNA demethylating agents and cell cycle checkpoint inhibitors may be promise therapies to breast cancers.

Immunophenopying has been proposed as an biological assay to classify tumors and their microenvironments ${ }^{[21-23]}$. A classification into inflamed and non-inflamed tumors, and presence of the immune cells, especially $\mathrm{T}$ cells, have been used as an indicator of clinical response to the immunotherapies ${ }^{[19-21]}$. The immune-inflamed phenotype is rich in immune cells responsive to the immune checkpoint monoclonal antibodies towards CTLA-4 and PD-1, as well as its ligands PD-L1 and PDL2. These mAbs are highly effective and specific, however, the main drawback of immunotherapies is heterogeneity of response rate, estimated from $10 \%-40 \%{ }^{[64]}$. CD $8+$ T-cell infiltration increases significantly with tumor mutation load ${ }^{[64]}$. The presence of both PD-L1 expression on breast tumor cells and TILs in TME predict longer disease-free survival and better overall survival to TNBC patients ${ }^{[65]}$. One study investigated the role of PD-1 as a prognostic marker for TNBC in an Asian cohort of 269 patients ${ }^{[66]}$. The results suggested a superior prognostic value of the CD274 (PDCD1 ligand 1, PD-L1) and PDCD1 in TNBC tumor immune microenvironment as compared to classical clinicopathological parameters ${ }^{[6]}$. Overall the results of these studies are providing new insights and alternative routes for possible therapeutic interventions to breast cancers.

An increased number of commensal and pathogenic bacteria, including Fusobacterium nucleatum, Bacteroides fragilis, Enterococcus faecalis, Streptococcus gallolyticus, Helicobacter hepaticus, and Porphyromonas gingivalis have been associated with cancer development. On the other hand, Faecalibacterium prausnitzii, Akkermansia muciniphila, Bifidobacterium longum, Collinsella aerofaciens and Citrobacter rodentium seem 
to have an anti-inflammatory and anti-tumoral roles in a variety of tumors ${ }^{[67]}$. A comprehensive analysis of microbiota of 668 breast tumors indicated that H. influenza and Listeria spp inhabiting the stromal breast cancer tissue significantly influence the expression of genes in the proliferative pathways: G2M checkpoint, E2F TFs, and mitotic spindle assembly ${ }^{[68]}$. Additionally, one study identified that Lactobacillus fleischmannii was associated with epithelial mesenchymal transition ${ }^{[69]}$. Oral supplementation with A. muciniphila probiotic restore the efficacy of PD-1 blockade against epithelial tumors by increasing the recruitment of CCR9+CXCR3+CD4+ T lymphocytes in an interleukin-12-dependent manner ${ }^{[69]}$. With the approval of the first chemo- immunotherapy combination for metastatic $\mathrm{TNBC}^{[70]}$, we expect that future studies correlating traditionally clinicopathological parameters with additive predictive valor of commensal bacteria will find best cancer response signatures to combination chemotherapy and immunotherapy.

Breast cancer canonical risk factors include age at diagnosis, age at menarche, nulliparity, age at first birth, number of children, months of breastfeeding, race, body mass index, menopausal status, absorption, combined oral contraceptives, age at menopause, prior benign breast disease, and family history (BRCA 1 and 2 and PTEN mutations) of breast cancer ${ }^{[7]}$. The hypothesis that estrogens and estrogen metabolites are implicated in female breast cancer etiology and progression has been confirmed for luminal A and luminal $B$ breast cancer subtypes ${ }^{[7]}$. Environmental factors such as endocrine disruptors compounds (xenobiotics, pesticides and pollutants) may influence mammary and gut microbiota ${ }^{[72,73]}$. However, there is no data to support that a putative disease-causing microorganism or if altered microbial pattern of women could have a role in the development and progression of the breast cancer disease ${ }^{[72,73]}$. The molecular pathological epidemiology (MPE) is a new discipline for investigating specific risk estimative for endogenous and exogenous factors controlling the etiologic heterogeneity of carcinogenic process, as well as environmental and inherited factors leading to failures to pharmaceutical treatment or prevention ${ }^{[7,75]}$. MPE incorporates large multi-omic and epigenomic datasets to estimate the impact of genome-wide association studies on disease entity in population-based cohorts ${ }^{[74,75]}$. MPE methodologies can provide novel insights into mechanistic pathways of common diseases and contribution of medications (pharmaco-MPE), immune mediators, and microorganism (microbial-MPE) on their risk expectative ${ }^{[75]}$. In this way, standardized pathological methods for breast cancer molecular subtyping (whole-tissue specimens or TMA), antibodies for immunohistochemistry, and imaging digital analytical repertories will have great value to establish the multidisciplinary framework for MPE investigations on definitive biomarkers to subtype breast cancers and genotype-phenotype relationships.

\section{CONCLUSION}

Breast cancer is a highly heterogeneous disease with differences in histopathological and biological characteristics, variable prognoses, and response to therapy. All breast tumor subtypes display different types of clonal subpopulations and this intratumoural and metastatic heterogeneity contribute to different drug sensitivities and resistance characteristics. Since the first study by Sorlie and colleagues in 2003, knowledge about genetic, epigenetic and endogenous and exogenous factors associated with the five subtypes of breast cancers (Luminal A, Luminal B, HER2-enriched, Basal-like and Claudin-low) and their associated aberrant signaling pathways extensively increased and become extremely complex. The multiomics and MPE methods for large comprehensive molecular cataloguing of cancer patient cohorts will help to establish and predictive biomarkers essential for new therapeutic agents and patients' responses. A systematic stratification of tumors based on therapeutically actionable mutated gene, TME immune cell profile, mammary microbial profile and epigenetic functional signatures may better predict those patients that will benefit from (neo) adjvuvant multi-agent chemotherapy, targeted and combination therapies, including immunotherapies. 


\section{DECLARATIONS}

\section{Acknowledgments}

Maria Mitzi Brentani, Fiorita Mundim, Alda Wakamatsu, Venancio AF Alves (University of São Paulo School of Medicine Medicine) and Dr Victor Piana Andrade e Fernando Soares (AC Carmargo Cancer Center) for their help and fruitful discussions.

\section{Author's contribution}

Study conception: Belizario JE, Loggulo AF

Selection and critical review of the papers and manuscript preparation: Belizario JE, Loggulo AF

Read and approved the final manuscript: Belizario JE, Loggulo AF

\section{Availability of data and materials}

Not applicable.

\section{Financial support and sponsorship}

JEB is supported by fellowship from "Conselho Nacional de Desenvolvimento Científico e Tecnológico" (CNPq proc 486048/2011; 312206/2016-0).

\section{Conflicts of interest}

Both authors declared that there are no conflicts of interest.

\section{Ethical approval and consent to participate}

Not applicable.

\section{Consent for publication}

Not applicable.

\section{Copyright}

(c) The Author(s) 2019.

\section{REFERENCES}

1. Belizario JE. Cancer risks linked to the bad luck hypothesis and epigenomic mutational signatures. Epigenomes 2018;2:13.

2. Lawrence MS, Stojanov P, Mermel CH, Robinson JT, Garraway LA et al. Discovery and saturation analysis of cancer genes across 21 tumour types. Nature 2014;505:495-501.

3. Vasaikar SV, Straub P, Wang J, Zhang B. LinkedOmics: analyzing multi-omics data within and across 32 cancer types. Nucleic Acids Res 2018;46:D956-63.

4. Kim YA, Cho DY, Przytycka TM. Understanding genotype - phenotype effects in cancer via network approaches. PLoS Comput Biol 2016;12:e1004747.

5. Barretina J, Caponigro G, Stransky N, Venkatesan K, Margolin AA, et al. The Cancer Cell Line Encyclopedia enables predictive modeling of anticancer drug sensitivity. Nature 2012;483:603-7.

6. Garnett MJ, Edelman J, Heidorn SJ, Greenman CD, Dastur A, et al. Systematic identification of genomic markers of drug sensitivity in cancer cells. Nature 2012;483:570-5.

7. Klijn C, Durinck S, Stawiski EW, Haverty PM, Jiang Z, et al. A comprehensive transcriptional portrait of human cancer cell lines. Nat Biotechnol 2015;33:306-12.

8. Campbell J, Ryan CJ, Brough R, Bajrami I, Pemberton HN, et al. Large-scale profiling of kinase dependencies in cancer cell lines. Cell Rep 2016;15:14:2490-501.

9. Polyak K, Haviv I, Campbell IG. Co-evolution of tumor cells and their microenvironment. Trends Genet 2008;25:30-8.

10. McGranahan N, Swanton C. Biological and therapeutic impact of intratumor heterogeneity in cancer evolution. Cancer Cell 2015;27:15-26

11. Belizario JE, Sangiuliano BA, Perez-Sosa M, Neyra JM, Moreira DF. Using pharmacogenomic databases for discovering patienttarget genes and small molecule candidates to cancer therapy. Front Pharmacol 2016;7:312.

12. Feinberg AP, Koldobskiy MA, Göndör A. Epigenetic modulators, modifiers and mediators in cancer aetiology and progression. Nat 
Rev Genet 2016;17:284-99.

13. Roy DM, Walsh LA, Chan TA. Driver mutations of cancer epigenomes. Protein Cell 2014;5:265-96.

14. Jeggo PA, Pearl LH, Carr AM. DNA repair, genome stability and cancer: A historical perspective. Nat Rev Cancer 2016;16:35-42.

15. Stirzaker C, Taberlay PC, Statham AL, Clark SJ. Mining cancer methylomes: prospects and challenges. Trends Genet 2014;30:75-84.

16. Yao L, Shen H, Laird PW, Farnham PJ, Berman BP. Inferring regulatory element landscapes and transcription factor networks from cancer methylomes. Genome Biol. 2015;16:105.

17. Widschwendter M, Jones A, Evans I, Reisel D, Dillner J, et al. Epigenome-based cancer risk prediction: rationale, opportunities and challenges. Nat Rev Clin Oncol 2018;15:292-309.

18. Mahoney KM, Rennert PD, Freeman GJ. Combination cancer immunotherapy and new immunomodulatory targets. Nat Rev Drug Discov 2015;14:561-84.

19. Blank CU, Haanen JB, Ribas A, Schumacher TN. Cancer Immunology. The cancer Immunogram. Science 2016;352:658-60.

20. Dumont N, Liu B, DeFilippis RA, Chang H, Rabban JT. Breast fibroblasts modulate early dissemination, tumorigenesis, and metastasis through alteration of extracellular matrix characteristics. Neoplasia 2013;15:249.

21. Thorsson V, Gibbs DL, Brown SD, Wolf D, Bortone DS, et al. The immune landscape of cancer. Immunity 2018;48:812-30.

22. Wang JC, Dick JE. Cancer stem cells: lessons from leukemia. Trends Cell Biol 2005;15:494-501.

23. Park SY, Lee HE, Li H, Shipitsin M, Gelman R, et al. Heterogeneity for stem cell-related markers according to tumor subtype and histologic stage in breast cancer. Clin Cancer Res 2010;16:876-87.

24. Kalluri R, Weinberg RA. The basics of epithelial-mesenchymal transition. J Clin Invest 2009;119:1420-8.

25. Aparicio S, Mardis E. Tumor heterogeneity: next-generation sequencing enhances the view from pathologist's microscope. Genome Biol 2014;15:463.

26. Laskin J, Jones S, Aparicio S, Chia S, Ch'ng C, et al. Lessons learned from the application of whole-genome analysis to the treatment of patients with advanced cancers. Cold Spring Harb Mol Case Stud 2015;1:a000570.

27. Mertins P, Mani DR, Ruggles KV, Gillette MA, Clauser KR, et al. Proteogenomics connects somatic mutations to signaling in breast cancer. Nature 2016;534:55-62.

28. Guerin M, Gonçalves A, Toiron Y, Baudelet E, Audebert S, et al. How may targeted proteomics complement genomic data in breast cancer? Expert Rev Proteomics 2017;14:43-54.

29. Shipitsin M, Campbell LL, Argani P, Weremowicz S, Bloushtain-Qimron N, et al. Molecular definition of breast tumor heterogeneity. Cancer Cell 2007;11:259-73.

30. Reis-Filho J, Pusztai L. Gene expression profiling in breast cancer: classification, prognostication, and prediction. Lancet 2011;378:1812-23.

31. Gudjonsson T, Adriance MC, Sternlicht MD, Petersen OW, Bissel MJ. Myoepithelial cells: their origin and function in breast morphogenesis and neoplasia. J Mammary Gland Biol Neoplasia 2005;10:261-72.

32. Stephens PJ, Tarpey PS, Davies H, Van Loo P, Greenman C, et al. The landscape of cancer genes and mutational processes in breast cancer. Nature 2012;486:400-4.

33. Sorlie T, Tibshirani R, Parker J, Hastie H, Marron JS, et al. Repeated observation of breast tumor subtypes in independent gene expression data sets. Proc Natl Acad Sci U S A 2003;100:8418-23.

34. Curtis CSP, Shah SF, Chin G, Turashvili OM, Rueda MJ, et al. The genomic and transcriptomic architecture of 2,000 breast tumours reveals novel subgroups. Nature 2012;486:346-52.

35. Perou CM, Sørlie T, Eisen MB, van de Rijn M, Jeffrey SS, et al. Molecular portraits of human breast tumours. Nature 2000;406:74752.

36. De Mattos-Arruda L, Ng CKY, Piscuoglio S, Gonzalez-Cao M, Lim RS, et al. Genetic heterogeneity and actionable mutations in HER2-positive primary breast cancers and their brain metastases. Oncotarget 2018;9:20617-30.

37. Turner NC, Reis-Filho JS. Basal-like breast cancer and the BRCA1 phenotype. Oncogene 2006;25:5846-53.

38. Gonzalez-Angulo AM, Morales-Vasquez F, Hortobagyi GN. Overview of resistance to systemic therapy in patients with breast cancer. Adv Exp Med Biol 2007;608:1-22.

39. Razavi P, Chang MT, Xu G, Bandlamudi C, Ross DS, et al. The genomic landscape of endocrine-resistant advanced breast cancers. Cancer Cell 2018;34:427-38.

40. Arteaga CL, Sliwkowski MX, Osborne CK, Perez EA, Puglisi F, et al. Treatment of HER2-positive breast cancer: current status and future perspectives. Nat Rev Clin Oncol 2012;9:16-32.

41. Pareja F, Reis-Filho JS. Triple-negative breast cancers - a panoply of cancer types. Nat Rev Clin Oncol 2018;15:347-8.

42. Cancer Genome Atlas Network. Comprehensive molecular portraits of human breast tumours. Nature 2012;490:61-70.

43. Alexandrov LB, Nik-Zainal S, Wedge DC, Aparicio AS, Behjati S, et al. Signatures of mutational processes in human cancer. Nature 2013;500:415-21.

44. Mundim FG, Pasini FS, Nonogaki S, Rocha RM, Soares FA, et al. Breast carcinoma-associated fibroblasts share similar biomarker profiles in matched lymph node metastasis. Appl Immunohistochem Mol Morphol 2016;24:712-20.

45. Harris LN, Ismaila N, McShane LM, Andre F, Collyar DE, et al. Use of biomarkers to guide decisions on adjuvant systemic therapy for women with early-stage Invasive breast cancer: American Society of Clinical Oncology Clinical Practice Guideline. J Clin Oncol 2016;34:1134-50.

46. Weaver DL, Ashikaga T, Krag DN, Skelly JM, Anderson SJ, et al. Effect of occult metastases on survival in node-negative breast cancer. N Engl J Med 2011;364:412-21. 
47. Liu MC, Pitcher BN, Mardis ER, Davies SR, Friedman PN, et al. PAM50 gene signatures and breast cancer prognosis with adjuvant anthracycline- and taxane-based chemotherapy: correlative analysis of C9741 (Alliance). NPJ Breast Cancer 2016;2:15023.

48. Li WX, He K, Tang L, Dai SX, Li GH, et al. Comprehensive tissue-specific gene set enrichment analysis and transcription factor analysis of breast cancer by integrating 14 gene expression datasets. Oncotarget 2017;8:6775-86.

49. Bancovik J, Moreira D, Porter D, Carrasco D, Yao J, et al. Dermcidin exerts its oncogenic effects in breast cancer via modulation ERBB signaling. BMC Cancer 2015;15:70.

50. Wilhelm M, Schlegl J, Hahne H, Gholami AM, Lieberenz M, et al. Mass-spectrometry-based draft of the human proteome. Nature 2014;509:582-7.

51. Scaltriti M, Nuciforo P, Bradbury I, Sperinde J, Agbor-Tarh D, et al. High HER2 expression correlates with response to the combination of lapatinib and trastuzumab. J. Clin Cancer Res 2015;21:569-76.

52. Kirouac DC, Du J, Lahdenranta J, Onsum MD, Nielsen UB, et al. HER2+ cancer cell dependence on PI3K vs. MAPK signaling axes is determined by expression of EGFR, ERBB3 and CDKN1B. PLoS Comput Biol 2016;12:e1004827.

53. Osmanbeyoglu HU, Pelossof R, Bromberg JF, Leslie CS. Linking signaling pathways to transcriptional programs in breast cancer. Genome Res 2014;24:1869-80.

54. Osmanbeyoglu HU, Toska E, Chan C, Baselga J, Leslie CS. Pancancer modelling predicts the context-specific impact of somatic mutations on transcriptional programs. Nature Commun 2017;8:14249.

55. Fackler MJ, Umbricht CB, Williams D, Argani P, Cruz LA, et al. Genome-wide methylation analysis identifies genes specific to breast cancer hormone receptor status and risk of recurrence. Cancer Res 2011;71:6195-207.

56. Su Y, Subedee A, Bloushtain-Qimron N, Savova V, Krzystanek M, et al. Somatic cell fusions reveal extensive heterogeneity in basallike breast cancer. Cell Rep 2015;11:1549-63.

57. Droog M, Mensink M, Zwart W. The estrogen receptor $\alpha$-cistrome beyond breast cancer. Mol Endocrinol 2016;30:1046-58.

58. Mei S, Meyer CA, Zheng R, Qin Q, Wu Q, et al. Cistrome Cancer: a web resource for integrative gene regulation modeling in cancer. Cancer Res 2017;77:19-22

59. Fleischer T, Tekpli X, Mathelier A, Wang S, Nebdal D, et al. DNA methylation at enhancers identifies distinct breast cancer lineages. Nat Commun 2017;8:1379.

60. Garrido-Castro AC, Goel S. CDK4/6 Inhibition in breast cancer: mechanisms of response and treatment failure. Curr Breast Cancer Rep 2017;9:26-33.

61. Goel S, DeCristo MJ, Watt AC, BrinJones H, Sceneay J, et al. CDK4/6 inhibition triggers anti-tumour immunity. Nature 2017;548:471-5.

62. Fiegl H, Millinger S, Goebel G, Müller-Holzner E, Marth C, et al. Breast cancer DNA methylation profiles in cancer cells and tumor stroma: association with HER-2/neu status in primary breast cancer. Cancer Res 2006;66:29-33.

63. Costa A, Kieffer Y, Scholer-Dahirel A, Pelon F, Bourachot B, et al. Fibroblast heterogeneity and immunosuppressive environment in human breast cancer. Cancer Cell 2018;33:463-79.

64. Force J, Leal JHS, McArthur HL. Checkpoint blockade strategies in the treatment of breast cancer: where we are and where we are heading. Curr Treat Options Oncol 2019;20:35.

65. Mori H, Kubo M, Yamaguchi R, Nishimura R, Osako T, et al. The combination of PD-L1 expression and decreased tumor-infiltrating lymphocytes is associated with a poor prognosis in triple-negative breast cancer. Oncotarget 2017;8:15584-92.

66. Yeong J, Lim JCT, Lee B, Li H, Ong CCH, et al. Prognostic value of CD8+PD-1+ immune infiltrates and PDCD1 gene expression in triple negative breast cancer. J Immunother Cancer 2019;7:34.

67. Dzutsev A, Badger JH, Perez-Chanona E, Roy S, Salcedo R, et al. Microbes and cancer. Annu Rev Immunol 2017;35:199-228.

68. Thompson KJ, Ingle JN, Tang X, Chia N, Jeraldo PR, et al. A comprehensive analysis of breast cancer microbiota and host gene expression. PLoS One 2017;12:e0188873.

69. Routy B, Le Chatelier E, Derosa L, Duong CPM, Alou MT, et al. Gut microbiome influences efficacy of PD-1-based immunotherapy against epithelial tumors. Science 2018;359:91-7.

70. D’Abreo N, Adams S. Immune-checkpoint inhibition for metastatic triple-negative breast cancer: safety first? Nat Rev Clin Oncol 2019;16:399-400.

71. Rojas K, Stuckey A. Breast cancer epidemiology and risk factors. Clin Obstet Gynecol 2016;59:651-72.

72. Safe S, Li X. Endocrine disruption: relevance of experimental studies in female animals to human studies. Curr Opin Toxicol 2017;3:12-9.

73. Reid G. Can breast microbiota provide protective effects against cancer? Future Microbiol 2016;11:987-99.

74. Hamada T, Keum N, Nishihara R, Ogino S. Molecular pathological epidemiology: new developing frontiers of big data science to study etiologies and pathogenesis. J Gastroenterol 2017;52:265-75.

75. Ogino S, Nowak JA, Hamada T, Milner DA Jr, Nishihara R. Insights into pathogenic interactions among environment, host, and tumor at the crossroads of molecular pathology and epidemiology. Annu Rev Pathol 2019;14:83-103. 